\title{
BMJ Global Health The impact of the COVID-19 pandemic on the incidence and mortality of zoonotic diseases in China
}

\author{
Chenjin Ma (D) , ${ }^{1}$ Xiangyu Guo, ${ }^{2}$ Lan Wang, ${ }^{3}$ Wang Li, ${ }^{4}$ Shelan Liu, ${ }^{5}$ Feng Lin, ${ }^{5}$ \\ Wangli Xu (1) ${ }^{2}$
}

To cite: Ma C, Guo X, Wang $L$, et al. The impact of the COVID-19 pandemic on the incidence and mortality of zoonotic diseases in China. BMJ Global Health 2022;7:e007109. doi:10.1136/ bmjgh-2021-007109

Handling editor Seye Abimbola

- Additional supplemental material is published online only. To view, please visit the journal online (http://dx.doi.org/10. 1136/bmjgh-2021-007109).

$\mathrm{CM}, \mathrm{XG}$ and $\mathrm{LW}$ contributed equally.

Received 5 August 2021 Accepted 16 December 2021

Check for updates

(C) Author(s) (or their employer(s)) 2022. Re-use permitted under CC BY-NC. No commercial re-use. See rights and permissions. Published by BMJ.

For numbered affiliations see end of article.

\section{Correspondence to}

Professor Shelan Liu; liushelan@126.com,

Professor Feng Lin;

feng|@cdc.zj.cn and Professor

Wangli Xu;

wlxu@ruc.edu.cn

\section{ABSTRACT}

Background It has been reported that strict nonpharmaceutical measures can significantly reduce the incidence and mortality of respiratory and intestinal infectious diseases during the COVID-19 pandemic, but there are limited reports on the impact in terms of the rates of zoonotic diseases.

Methods We extracted the incidence and mortality data of eight notifiable infectious zoonotic diseases from the website of the National Health Commission of the People's Republic of China for the period of January 2015 to April 2021.

Results First, the overall incidence of zoonotic diseases decreased from 0.3714 per 100000 in 2015-2019 to 0.2756 in 2020 ( $25.79 \%$ reduction, $p<0.001)$; however, a dramatic increase in activity was seen in 2021 compared with 2020 (0.4478 per 100000 in 2021, 62.47\% increase, $\mathrm{p}<0.001)$. Anthrax, brucellosis, leptospirosis and hydatid disease exhibited significant upward trends in 2021. Second, analysed further by stages, the monthly incidence in the routine stage (from May to December 2020) was much higher than that in the emergency stage of the COVID-19 (from January to April 2020) (55.33\% increase, $p<0.001)$. We also found that the monthly observed incidence was significantly lower than the predicted incidence of a $10.29 \%$ reduction in the emergency stage. Third, no differences were seen in mortality between 2021 and 2020, while a significant decline was found in 2020 compared with the previous 5 years $(72.70 \%, p<0.001)$. Conclusions Strict containment and feasible suppression strategies during the 2020 period of the COVID-19 pandemic had positive impacts on the overall incidence of zoonotic diseases in China. However, anthrax, brucellosis, leptospirosis and hydatid diseases might increase with the relaxation of non-pharmacological interventions in 2021.

\section{INTRODUCTION}

Zoonotic diseases are infectious diseases that are vectorborne by pathogens in wild, synanthropic and domesticated host animals and can only infect people (accidental hosts) when they enter a natural focus. ${ }^{12}$ Zoonotic pathogens can be bacterial, viral or parasitic, and may involve unconventional agents, spreading to humans through direct contact or through

\section{Key questions}

What is already known?

- COVID-19 and zoonotic diseases are two of the most challenging public health problems.

- Strict non-pharmacological interventions have been shown to significantly reduce the incidence and mortality of respiratory and intestinal infectious diseases throughout the COVID-19 pandemic.

\section{What are the new findings?}

- The average monthly incidence of total zoonotic diseases from January to April 2021 was significantly higher than that in 2020 and in 2015-2019. These increases may have been caused by brucellosis.

- There was no significant difference in monthly mortality between the emergency stage and the routine stage of the COVID-19 pandemic in 2020.

- The observed monthly incidence rate during the emergency stage of COVID-19 in 2020 was significantly lower than that predicted.

\section{What do the new findings imply?}

- Strict containment and suppression strategies in the emergency stage of the COVID-19 pandemic had a significant impact on reducing the incidence of zoonotic diseases.

- The impact of mild non-pharmaceutical routine measures on zoonotic diseases in the postpandemic era was minimal.

food, water or the environment. ${ }^{3}$ Over the past two decades, views of and research on the diversity, spread and epidemic characteristics of zoonotic diseases have been proposed worldwide. Some epidemiological studies have confirmed that about $61 \%,{ }^{4}$ even $75 \%$, of the total number of pathogens affecting humans are zoonotic. Moreover, increases in the scope of human activity (such as the expansion of urban construction) have led to a significant increase in contact between humans and the natural focus, creating favourable epidemiological transmission for zoonotic diseases. ${ }^{16}$ Such increased risk of exposure to pathogens may lead to clusters 
of cases or even to larger outbreaks. Zoonotic diseases constitute a serious potential threat to worldwide public health. In China, as in the rest of the world, zoonotic diseases are serious and cannot be ignored. According to national data, current cases of zoonotic diseases account for $60 \%$ of all infectious diseases in China, and the annual death rate due to zoonotic diseases makes up 4.3\%-1.4\% of the total number of deaths due to infectious diseases. ${ }^{7}$

COVID-19 is likely to be the most disastrous infectious disease outbreak in decades and was characterised as a pandemic by the WHO in March 2020. ${ }^{8}$ The first major outbreak occurred in the city of Wuhan in the Hubei province of China. COVID-19 has rapidly spread to more than 225 countries, areas or territories, and as of 12 November 2021, a total of 251 million confirmed cases have been reported worldwide. Meanwhile, genetic variants of the SARS-CoV-2 virus have been emerging and circulating around the world such as the B.1.617.2 (Delta) variant, which could cause a devastating new wave of infections due to its stronger transmission potential and possible evasion of immunity. ${ }^{9}$ To limit and control the spread of the virus, the local government indicated a series of nonpharmacological interventions (NPIs), including wearing face masks, more frequent hand washing, stay-at-home orders, ceasing travel and social distancing, with some cities going into lockdown. Multiple measures to restrict flow or access have also been imposed in public places. On the other hand, some governments have formulated animal-related measures to reduce contact with wild animals; for example, the government of Denmark culled 15 million minks in Danish farms to minimise the risk of retransmitting the new coronavirus to humans. In China, strict containment and suppression strategies, such as stay-at-home orders, ceasing travel and maintaining social distancing, were applied in the early emergency stage of the COVID-19 pandemic (January to April 2020) to control outbreaks and community spread. Thereafter, feasible and continuous routine prevention and control measures, such as wearing face masks and health codes, were mandated to keep cases down while allowing people to return to work and prepandemic everyday activities.

Such tough situations have changed people's lifestyles, ${ }^{10}{ }^{11}$ economic and environmental vectors, as well as the epidemic characteristics of other diseases. Some studies have reported a significant impact on respiratory conditions, ${ }^{12}{ }^{13}$ in particular seasonal influenza. Efforts to control the spread of the coronavirus led to decreased COVID-19 cases and to lower levels of potential transmission of other infectious diseases. For example, decreased influenza incidence has been reported worldwide, such as in the USA, Australia, Chile, South Africa, ${ }^{14}$ Singapore ${ }^{15}$ and Korea. ${ }^{13}$ On the other hand, some studies on lowincome and middle-income countries have also reported that strict movement restrictions might have caused delays in the diagnosis and treatment of other diseases, such as diseases caused by parasites, fungi, bacteria and viruses (eg, HIV, tuberculosis and malaria). ${ }^{16} 17$ Meanwhile, there is a rising worldwide trend that patients with non-COVID-19 emergency conditions are avoiding seeking medical care. ${ }^{18-20}$ Some patients have reported avoiding hospitals for fear of contracting severe coronavirus. Such changes may affect the epidemic characteristics of other diseases. For example, Xiao et al reported that compared with the synchronous period during 2015-2019, the number of notifiable infectious disease cases in Guangdong province, China, during the emergency response period in 2020 had decreased by $50.7 \%$. Among 39 types of notifiable infectious diseases, zoonotic diseases and insectborne infectious diseases had the greatest reduction $(89.4 \%)$, followed by respiratory infectious diseases $(87.4 \%)$, intestinal infectious diseases (59.4\%) and bloodborne and sexually transmitted infections $(18.2 \%) .{ }^{21}$ Finally, all NPIs implemented to control COVID-19, as discussed above, also limit human movements and reduce exposure risk to vectors, but the quality of this influence has not yet been well studied.

In this study, we focused on the impact of the COVID-19 pandemic on zoonotic diseases. COVID-19 and zoonotic diseases are two of the most challenging public health problems; therefore, it is necessary to investigate the impact of COVID-19 on the epidemic trends of zoonotic diseases. The goal of this study was to quantify the impact of the COVID-19 pandemic on zoonotic diseases in China. Notably, the number of confirmed and suspected cases of COVID-19 in China has been dramatically decreasing, and there has been no serious ongoing community transmission or surge in local cases reported for an extended period (except for internationally imported cases). Thus, the data obtained in China are an ideal source for examining the impact of COVID-19 from the emergency pandemic era into the present.

\section{METHODS}

\section{Data sources}

Since 2004, China has used the direct network reporting system called the China Infectious Disease Reporting Information System. A total of 40 notifiable infectious diseases must be reported compulsorily, including 10 kinds of zoonotic diseases, namely plague, H5N1, H7N9, haemorrhagic fever, rabies, anthrax, brucellosis, leptospirosis, hydatid disease and schistosomiasis.

Data on monthly cases of and deaths from 10 zoonotic diseases that occurred from January 2015 to April 2021 were obtained from the National Health Commission of the People's Republic of China, which releases a monthly report of notifiable infectious diseases online. ${ }^{22}$ Plague and $\mathrm{H} 5 \mathrm{~N} 1$ were removed from the present analysis because their sample sizes were too small to generate sensible estimates. Lastly, we extracted a total of eight kinds of zoonotic diseases (H7N9, haemorrhagic fever, rabies, anthrax, brucellosis, leptospirosis, hydatid disease and schistosomiasis). Seven of them are class B (H7N9, haemorrhagic fever, rabies, anthrax, brucellosis, leptospirosis and schistosomiasis), and one is class $\mathrm{C}$ (hydatid disease). The Chinese population data from January 
2015 to April 2021 were obtained from the National Bureau of Statistics. ${ }^{23}$ It should be noted that the cases of and deaths from zoonotic diseases included local and imported cases, of which most cases were local, particularly during the COVID-19 period.

\section{COVID-19 response strategies in China}

After the official declaration of the COVID-19 epidemic, China began to take comprehensive approaches to infectious disease control. On 22 January 2020, Hubei province took the lead in launching a second-level public health emergency response mechanism. Wuhan, the epicentre of the outbreak in China, has been on lockdown since 23 January. After the outbreak, all 31 provinces across China launched a first-level response. Detailed information about the start and end times of the public health emergency response mechanism in each province is presented in online supplemental table S1.

Containment and suppression strategies were applied in the early stages of the epidemic in some well-defined areas and when the epidemic was in multiple areas with varying degrees of outbreak and community spread. The core measures in some high-risk areas were lockdown of endemic areas, restricted travel from endemic areas to other low epidemic areas, stay-at-home orders, school closures, cancellations of mass gatherings, hand hygiene, respiratory etiquette and face mask use. Due to the effective implementation of multiple measures against the COVID-19 epidemic, new cases across China have shown a dramatic decline. Therefore, since 24 February 2020, according to the local situation of COVID-19, many provinces have successively adjusted the emergency response level from the first level to the second level, with some even moving to the third level. Since 2 May 2020, the emergency response level of public health emergency in Hubei province has been adjusted from a first-level response to a second-level response. Lockdown measures eased in Wuhan from 8 April 2020. So far, all provinces across China have lifted the first-level response. Measures under the China public health emergency response system are listed in online supplemental table S4.

After transmission was controlled and eliminated, the intensity of public health interventions was progressively relaxed, and the public health emergency response was lifted throughout Chinese provinces. A number of small outbreaks in a few cities were rapidly controlled through locally intense public health measures. COVID-19 surveillance and control measures continue in China through intensive tracing, isolation and contact measures to reduce the local incidence to zero ('Zero COVID-19' strategy). To compare the incidence and mortality of zoonotic diseases between the COVID-19 pandemic and postpandemic periods in this study, according to the Chinese government's division of the COVID-19 stages, the period from January 2020 to April 2020 was identified as the emergency stage of
COVID-19 in China, and the period from May 2020 to December 2020 was identified as the routine stage of COVID-19.

\section{Statistical analysis}

In the data analysis, summary statistics were applied. Specifically, for each zoonotic disease, the following averages were computed: annual incidence, annual number of cases, annual mortality, annual number of deaths, monthly incidence and monthly mortality. Comparisons across groups were made using two proportional tests and two ratio $\mathrm{Z}$ tests. Growth rate was computed to examine the growth of incidence and mortality. For example, in the comparison between 2020 and 2015-2019, the growth rate of monthly incidence was defined as follows:

$\frac{\text { monthly incidence in } 2020-\text { monthly incidence from } 2015 \text { to } 2019}{\text { monthly incidence from } 2015 \text { to } 2019} * 100$

The Cox-Stuart test was applied to assess whether there was an increasing or decreasing long-term trend of the monthly incidence and mortality from 2015 to 2019. If a disease was tested for an insignificant trend using the Cox-Stuart test, its incidence decline was due to COVID-19-related measures.

To avoid the interference of the long-term trend of disease itself, for each disease, the monthly predictive incidence from January to December 2020 was generated using Farrington surveillance algorithms to model the monthly incidence observed during 2015-2019. The Farrington surveillance algorithm has proved to be sensitive and reliable for treating trends, seasonality, error structure and the influence of past outbreaks. ${ }^{24}$ Thus, the significant difference between predicted value and observed value could be due to COVID-19related measures. This algorithm uses discrete Poisson generalised linear models with spline terms to estimate the trend in monthly incidence and is implemented by the surveillance package in $\mathrm{R}$. We also computed the lower and upper $95 \%$ CIs. In all tests, a p value cut-off of 0.05 was used.

\section{Patient and public involvement}

There was no patient or public involvement in this research.

\section{RESULTS \\ Overall review}

For incident cases, from January to April 2021, 2020 and 2015-2019, a total of 25016 cases, 63226 cases and 77448 cases (per year) of eight zoonotic diseases were reported, respectively. Among the eight types of zoonotic diseases, from January to April 2021, the top three diseases with the most cases were brucellosis $(86.62 \%)$, haemorrhagic fever $(7.44 \%)$ and hydatid disease $(5.34 \%)$. In 2020 , the three most common zoonotic diseases were brucellosis $(79.26 \%)$, haemorrhagic fever $(13.52 \%)$ and hydatid disease $(5.91 \%)$, which together accounted for up to $98.69 \%$ of all cases. 
From 2015 to 2019, the top three diseases with the largest numbers of cases were brucellosis (61.90\%), schistosomiasis $(15.56 \%)$ and haemorrhagic fever $(14.03 \%)$.

For fatal cases, 77 deaths, 244 deaths and 673 deaths (per year) were reported from January to April 2021, 2020 and 2015-2019, respectively. Among the eight types of zoonotic diseases, from January to April 2021, the disease with the largest number of deaths was rabies
$(88.31 \%)$. In 2020, rabies $(73.36 \%)$ and H7N9 $(20.90 \%)$ had the largest numbers of deaths. From 2015 to 2019, rabies $(76.62 \%)$, H7N9 $(12.76 \%)$ and haemorrhagic fever $(9.43 \%)$ had the largest numbers of deaths.

\section{Incidence in 2021, 2020 and 2015-2019}

The upper-left and lower-left panels in figure 1 show the monthly incidence trends for the eight zoonotic diseases from 2015 to April 2021. As clearly established in the
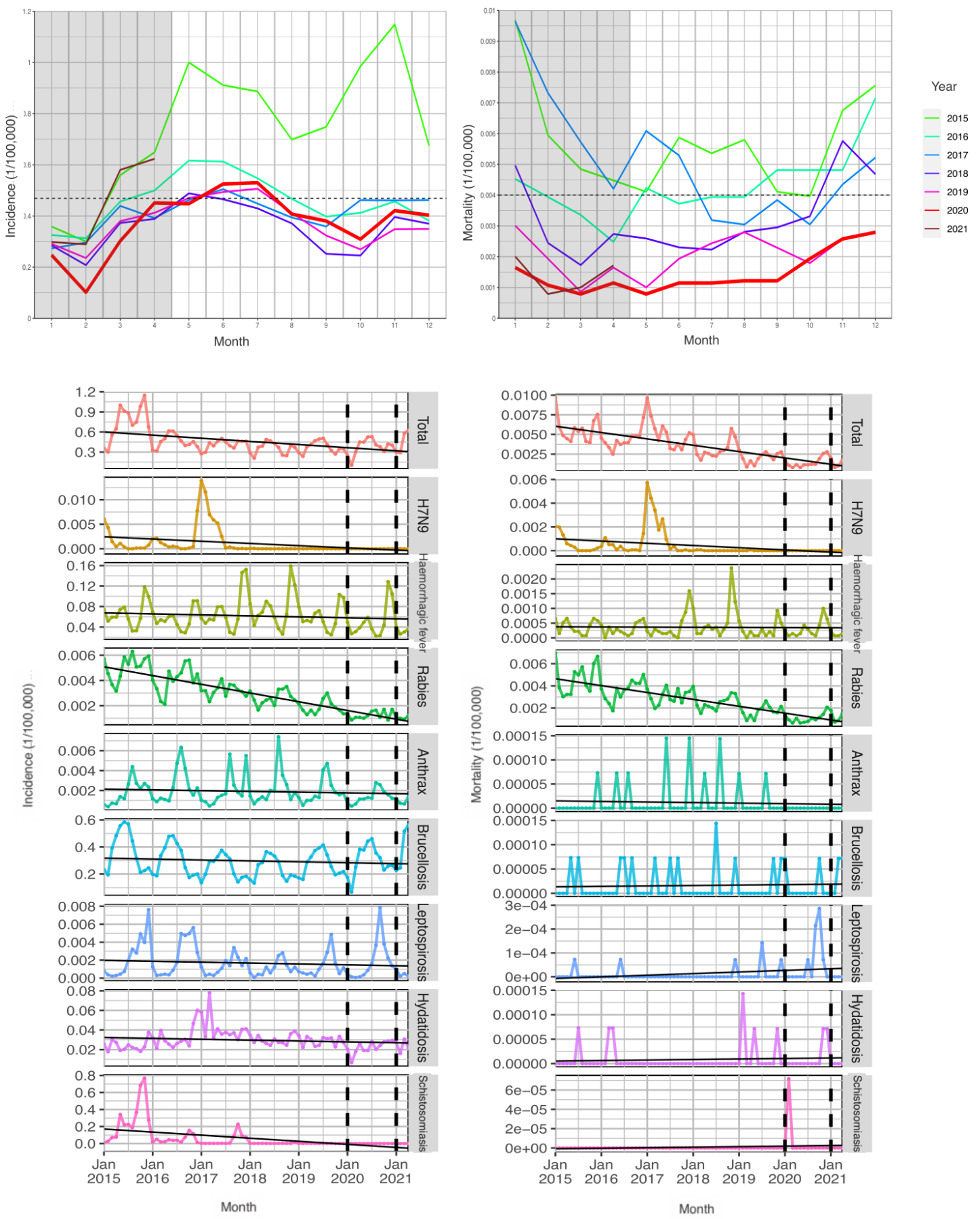

Figure 1 Trends of monthly incidence (upper-left panel) and monthly mortality (upper-right panel) of overall zoonotic disease in China from 2015 to April 2021. Trends of monthly incidence (lower-left panel) and the monthly mortality (lower-right panel) of zoonotic disease in China from 2015 to April 2021. The horizontal dotted lines in upper panel represent the average monthly incidence and mortality from 2015 to 2019. The black lines in the lower panel indicate the non-parametric fits. 
literature, zoonotic diseases have seasonality and longterm trends. From 2014 to 2020, 2015 had the highest incidence of such diseases. The incidence was relatively high between April and December each year. After entering the routine stage in 2020 , the incidence trend rose toward the average monthly incidence level during 2015-2019.

Combined with table 1, which presents the results of comparisons of average monthly incidence during January to April 2021, 2020 and 2015-2019, we found that, on the whole, the average monthly incidence of zoonotic diseases from January to April 2021 was both significantly higher than that in $2020(0.4478 / 100000$ vs $0.2756 / 100000$, growth rate: $62.47, \mathrm{p}<0.001)$ and 2015-2019 (0.4478/100 000 vs $0.3714 / 100000$, growth rate: $20.58, \mathrm{p}<0.001)$. The average monthly incidence of zoonotic diseases in 2020 was significantly lower than that in $2015-2019(0.2756 / 100000$ vs $0.3714 / 100000$, growth rate: $-25.79, \mathrm{p}<0.001)$. Specifically, brucellosis and hydatid disease had a higher monthly incidence throughout January to April 2021 than in 2020. Except for anthrax, leptospirosis and brucellosis from January to April 2021, the monthly incidences in January to April 2021 were significantly lower than those in 2020 and 2015.

Table 1 also shows the results of the trend analyses. For monthly incidence, there were significant long-term downward trends of total zoonotic diseases $(\mathrm{p}=0.02)$, H7N9 $(\mathrm{p}<0.001)$, rabies $(\mathrm{p}<0.001)$ and schistosomiasis $(p<0.001)$. It should be noted that, combined with the results of the trend analyses, the significant growth rate of some diseases (marked with *) might be due to their own long-term trends and/or the measures for controlling the COVID-19 pandemic.

\section{Mortality in 2021, 2020 and 2015-2019}

The upper-right and lower-right panels in figure 1 show the monthly mortality trends for the eight zoonotic diseases from 2015 to April 2021. The annual mortality had a long-term trend of decline with time. Monthly mortality continued to fall in 2020, far below the average of previous years. Monthly incidence trends and mortality trends for each disease are also presented in online supplemental figure S1.

As shown in table 1 and the lower-right panel in figure 1 combined, we found that the average monthly mortality of zoonotic diseases during January to April 2021 and 2020 was both significantly lower than the data reported for the same period in 2015-2019 $(0.00138 / 100$ 000 vs $0.00426 / 100000$, growth rate: $-67.66, \mathrm{p}<0.001$; $0.00116 / 100000$ vs $0.00426 / 100 \quad 000$, growth rate: $-72.70, \mathrm{p}<0.001)$. For monthly mortality, only H7N9 and rabies during January to April 2021 and 2020 had lower values than those in 2015-2019.

Furthermore, significant long-term downward trends were observed in terms of monthly mortality in total zoonotic diseases $(\mathrm{p}<0.001), \mathrm{H} 7 \mathrm{~N} 9(\mathrm{p}<0.001)$ and rabies $(\mathrm{p}<0.001)$ (table 1$)$. It should be noted that, combined with the results of the trend analyses, the significant growth rates of some diseases (marked with $\dagger$ ) might be due to their own long-term trends and/or the measures for controlling the COVID-19 pandemic. Summary of the annual incidence and mortality of zoonotic diseases has been presented in the online supplemental table S2.

\section{Incidence and mortality between emergency and routine stages}

Table 2 presents the results of the comparisons of average monthly incidence and average monthly mortality in 2020 and 2015-2019. Compared with the same period in 2015-2019, we found that in the emergency and routine stages of the COVID-19 pandemic, zoonotic diseases showed significant declines of $25.79 \%(0.2756 / 100000$ vs $0.3714 / 100000, \mathrm{p}<0.001)$ and $17.02 \%(0.4281 / 100$ 000 vs $0.5160 / 100000, \mathrm{p}<0.001)$, respectively.

For mortality, the significant declines in the growth rate during the emergency and routine stages of the COVID-19 pandemic were $72.39 \%(0.0012 / 100000$ vs $0.0043 / 100000, \mathrm{p}<0.001)$ and $59.30 \%(0.0016 / 100000$ vs $0.0040 / 100000, \mathrm{p}<0.001)$. Compared with the emergency stage, a significant increase was also found in the average monthly incidence in the routine stage $(0.4281 / 100000$ vs $0.2756 / 100000$, growth rate: $-55.33 \%, \mathrm{p}<0.001)$.

\section{Observed and predicted monthly incidence and mortality}

Observed and predicted monthly incidence and mortality are provided in figure 2 and table 3 . We found that for total zoonotic diseases, the observed monthly incidence in the emergency stage of 2020 was significantly lower than that predicted for the same period (growth rate $=-10.29 \%$, $\mathrm{p}<0.001)$. A significantly lower incidence was also observed in haemorrhagic fever (growth rate $=-17.05 \%$, $\mathrm{p}<0.001$ ) and brucellosis (growth rate $=-8.71 \%, \mathrm{p}<0.001$ )

The observed monthly incidence of brucellosis was significantly higher than that predicted for the same period (growth rate $=7.06 \%, \mathrm{p}=0.001$ ). No significant difference was found in mortality in either the emergency or routine stages. The results of the comparisons of the observed and predicted annual incidence and mortality are also presented in online supplemental table S3.

\section{DISCUSSION}

We reported the latest trends of eight zoonotic diseases in China during 2015-2021, with the largest sample size coving 31 provinces. We found a marked decline in the overall incidence rates of the eight zoonotic diseases in 2020 compared with the previous 5 years, but a dramatic increase in activity was seen in 2021 compared with 2020. In particular, anthrax, brucellosis, leptospirosis and hydatid disease exhibited significant upward trends in 2021. We analysed further by stages, and the monthly incidences in the routine stage (from May to December 2020) were much higher than those in the emergency stage (from January to April 2020). After removing the interference of long-term trends, the observed monthly incidence of zoonotic diseases in the emergency stage 


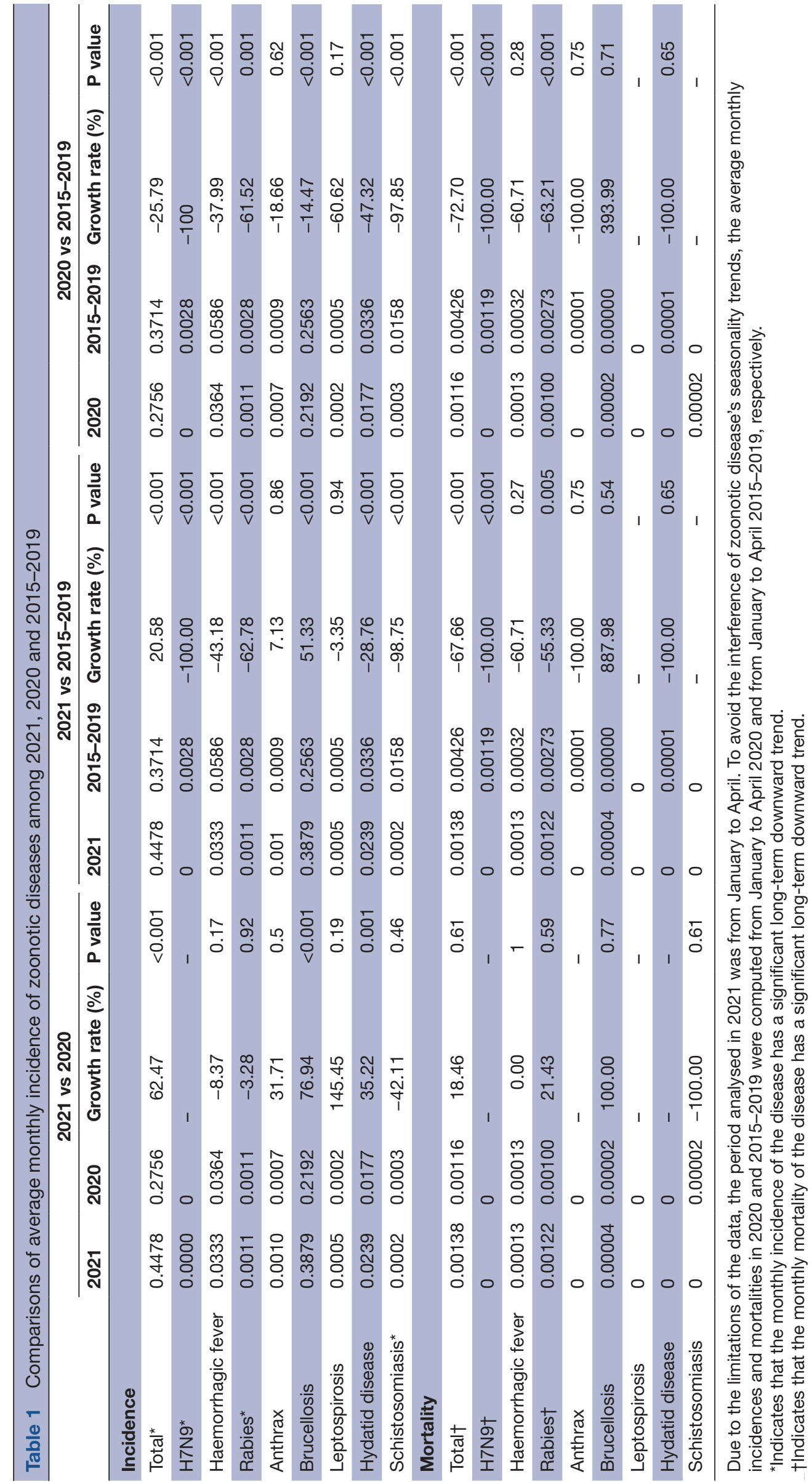




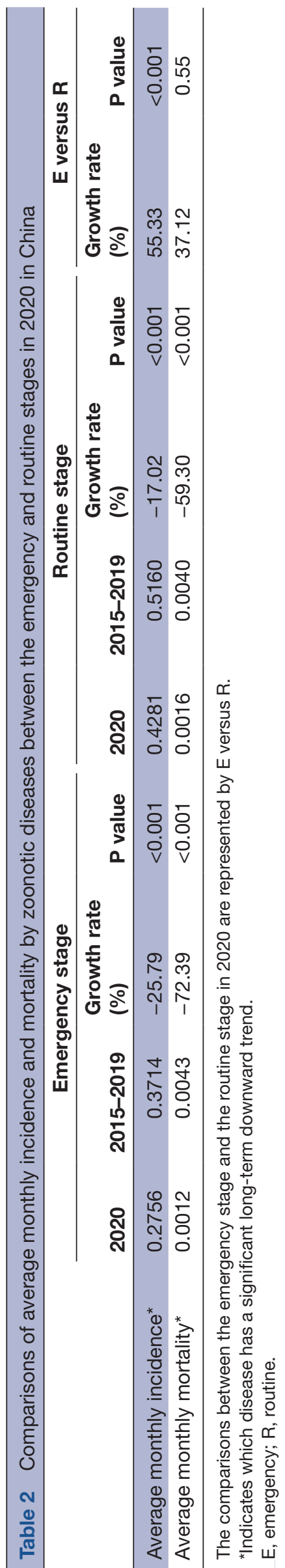

was significantly lower than the predicted incidence. For mortality, no differences were seen between 2021 and 2020, while a significant decline was found in 2020 compared with the previous 5 years.

For all zoonotic diseases, the average monthly incidence and mortality decreased over time, particularly in 2020, which could have been due to the following reasons. First, the non-pharmaceutical measures implemented during the emergency period of COVID-19 could have impacted this decrease. After the outbreak of COVID19 , strict containment and suppression strategies in the emergency stage (such as staying home, ceasing travel and maintaining social distancing) and mild preventive measures in the routine stage (such as wearing face masks, more frequent hand washing and the use of health $\mathrm{QR}$ codes) were implemented to control COVID-19, which also limited human movements and reduced human exposure to risk vectors. Second, the general avoidance of seeking medical care might also have contributed to such changes in the incidence of zoonotic diseases. As indicated above, some patients avoided hospitals for fear of contracting severe coronavirus. Especially in China, complex admission procedures during the COVID-19 period, such as going to fever clinics first to screen for COVID-19, undergoing the PCR tests for COVID-19 and presenting health $\mathrm{QR}$ codes, have also become obstacles to seeking medical care. This may lead to a decrease in cases of zoonotic diseases being recorded but will ultimately delay treatment, aggravate patients' conditions and potentially increase mortality rates. Third, during the COVID-19 pandemic, the build-up of health consciousness and large-scale disinfection have been organised by local governments, especially in rural areas and pasturing regions. In the early stages of the epidemic, the relevant traceability work of COVID-19 suggested that bats might be the hosts of this novel coronavirus. With the spread of this information and the related public broadcasts from the health department within China, people likely had greater awareness regarding avoiding contact with wild animals. The killing of possible host animals that may be at risk, such as livestock, poultry and rodents with potential zoonotic pathogens, also reduced contact between natural animals and people, and thus reduced exposure risk. Fourth, some vaccination programmes have had a positive impact on the incidence and mortality of zoonotic diseases. For example, the nationwide vaccination of chickens with an $\mathrm{H} 5 / \mathrm{H} 7$ bivalent inactivated avian influenza vaccine since September 2017 in China has successfully controlled H7N9 avian influenza infections in poultry. This vaccination programme has also prevented human infections. ${ }^{25}$ The impact of such vaccines has been reported in other countries as well. Raynor et al reported that the yearly mass dog vaccination programme in Peru was interrupted during COVID19 , resulting in a negative effect on rabies elimination and prevention activities. ${ }^{26}$ Fifth, decreased incidence and mortality of zoonotic diseases might be due to their own long-term downward trends, which is a confounding 

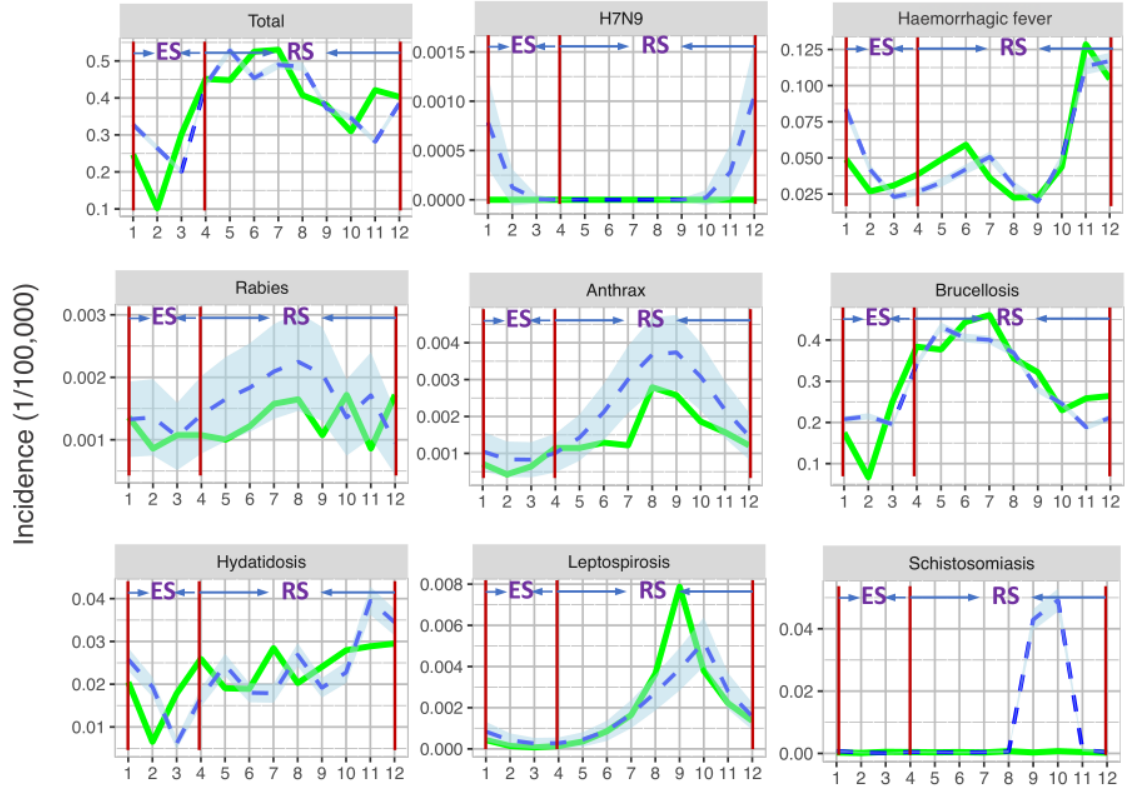

Month
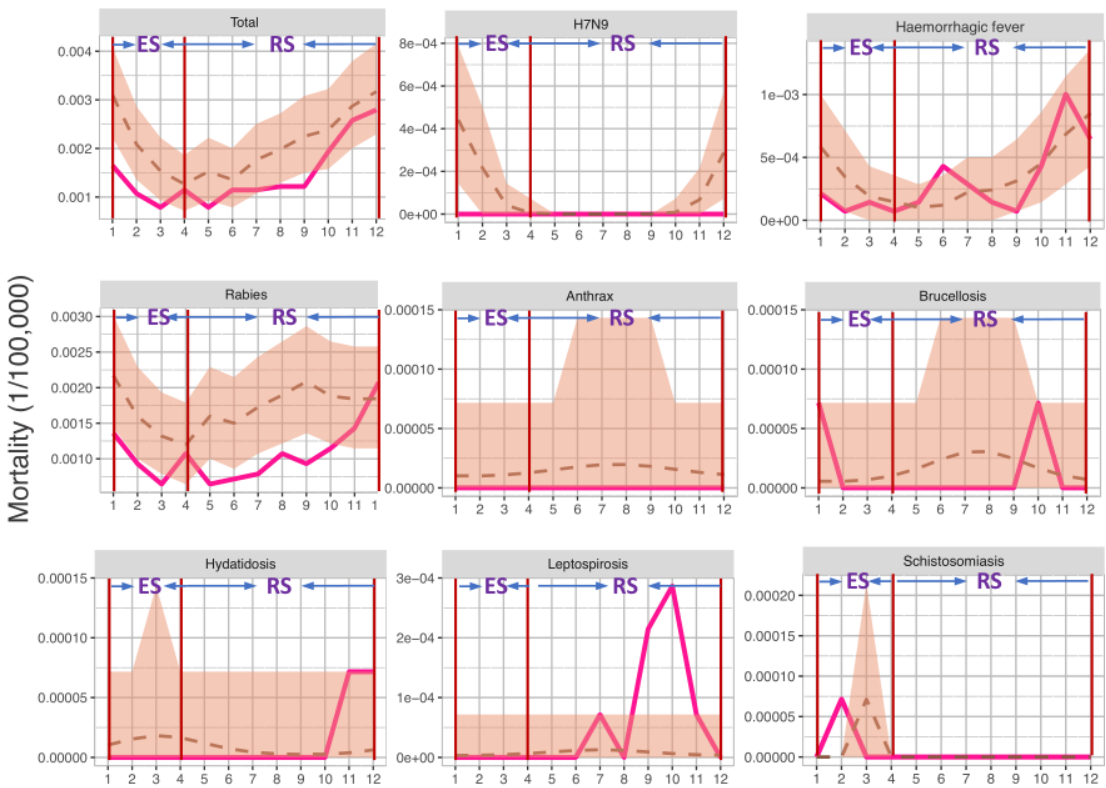

Month

Figure 2 Predictive trends (blue dotted lines) and observed trends (green lines) of the monthly incidence (upper panel) and monthly mortality (lower panel) in 2020. The light shaded areas are 95\% Cls. ES, emergency stage; RS, route stage.

factor in this study. However, to avoid the interference of the long-term trend of disease itself, some analyses were conducted, including the Cox-Stuart test and Farrington surveillance algorithm. Especially the Farrington surveillance algorithm, based on the advantages of this method, the significant difference between predicted incidence and observed incidence could be due to COVID-19related measures. It should be noted that the vaccination programmes discussed in the fourth point were one of the reasons for the long-term downward trends. Under non-strict conditions, the third point could be broadly considered as the non-pharmaceutical measures, and the second point could be considered as the one result of the non-pharmaceutical measures. Finally, regarding mortality, medical diagnosis and discovery capabilities have increased, particularly since the emergency stage, and thus the early detection of cases with respiratory symptoms similar to COVID-19 is now possible, resulting in a significant reduction in deaths.

In our results, except for the monthly incidence of brucellosis and schistosomiasis, observed incidence and mortality during the routine stage in 2020 were insignificantly different than predicted. Such findings suggest that the measures to prevent COVID-19 in the routine stage, especially wearing face masks, did not have any significant impact on the monthly incidence and mortality of 


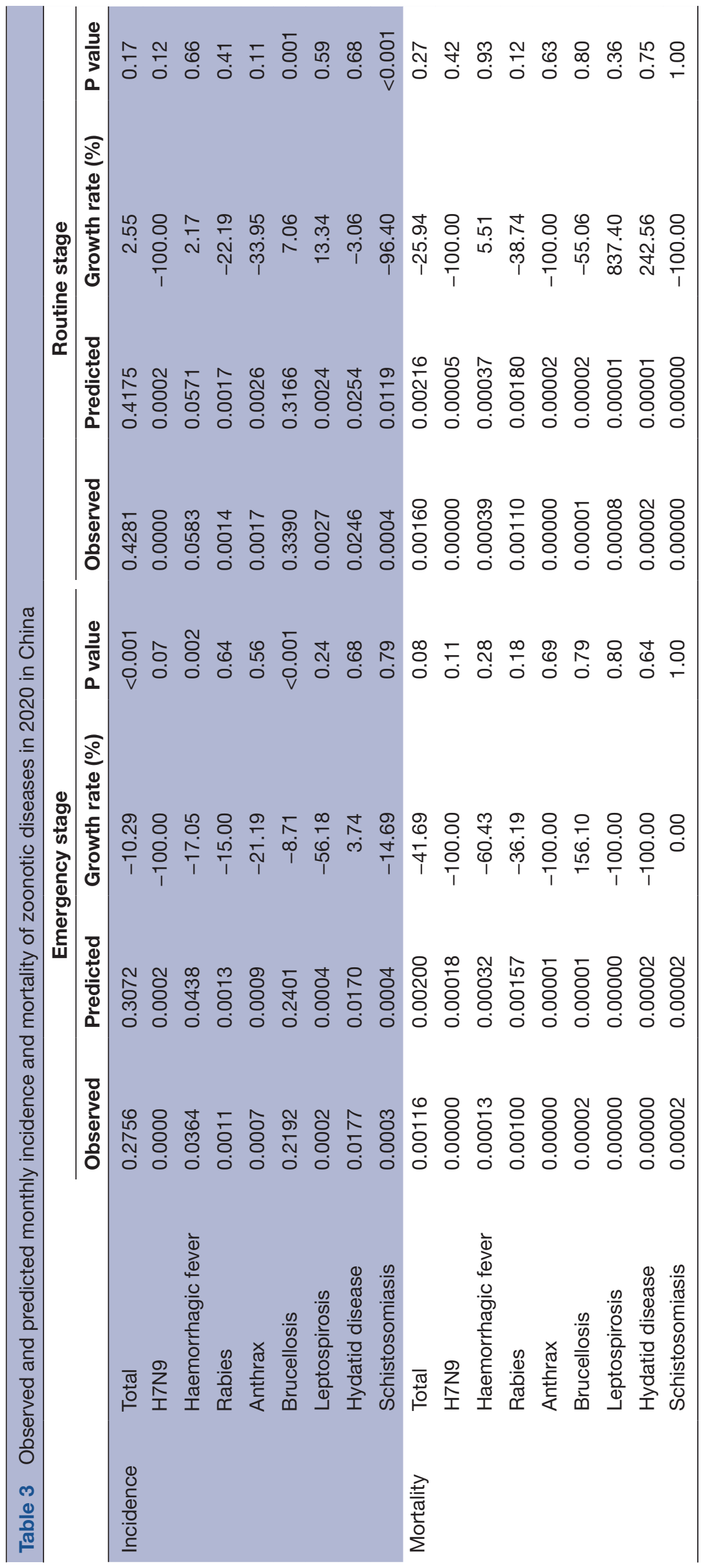

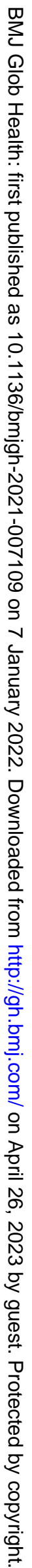


zoonotic diseases. In the routine stage of COVID-19, society has begun to gradually recover from stagnation of the economy, production, consumption and tourism. Movement restrictions have been removed, and the only remaining prevention and control measures against COVID-19 across China are wearing masks, using health QR codes and having PCR testing for COVID-19 done if necessary. The cough or sneeze of an infected person is the primary way COVID-19 is spread. Surgical masks can thus prevent the transmission of the COVID-19 virus from people who are infected. ${ }^{27} 28$ This is in contrast to zoonotic diseases, where getting into a natural focus and contacting the infectious vector or the reservoir host are the main transmissions of zoonotic diseases, not coughs or sneezes. Thus, for most zoonotic diseases, the effectiveness of the preventive measures employed during the routine stage of the COVID-19 pandemic to protect people from being infected and dying of zoonotic diseases is thought to be limited.

\section{Special cases}

Some special cases were found in the present study. As shown in table 1, more attention should be paid to brucellosis, as only it had a significantly positive growth rate in 2021 and 2020 among the eight zoonotic diseases. The monthly incidence of brucellosis from January to April 2021 was significantly higher than in other periods. The areas with rising cases were mainly concentrated in several areas in the north of China, where sheep trading and slaughter were more pronounced. This sheep trading and slaughter were stopped during the COVID-19 pandemic, but the cases of brucellosis among individual farmers and within breeding circulation units have increased due to the lack of improvement in the corresponding prevention measures.

During the emergency stage of the COVID-19 pandemic, the monthly incidences of haemorrhagic fever and brucellosis were significantly lower than those during the same period in 2015-2019. These significant differences were the main contributors to the significantly lower observed incidences of the total zoonotic diseases. It should be noted that these two diseases, brucellosis and haemorrhagic fever, had the largest number of cases, ranking first and second in 2020, and ranking first and third during 2015-2019. The main reason for the decrease in overall incidence during the emergency stage was the rapid activation of a public health emergency response after the outbreak of COVID-19. Correspondingly, various prevention and control measures limited population mobility and restricted human activity, which could have reduced epidemiological transmission between humans and the natural focus of zoonotic diseases.

We found that the monthly incidence of other zoonotic diseases (H7N9, rabies, anthrax, leptospirosis, hydatid disease and schistosomiasis) during the emergency stage was not sensitive to the measures enacted for controlling the COVID-19 pandemic. This finding might be due to the long-term prevention and control of these diseases, which led to a decreasing number of cases year by year. Especially in recent years, the annual number of cases of zoonotic diseases has been very small and relatively stable; for example, H7N9 had annual case numbers of 204, 216, $646,2,1$ and 0 in the years from 2015 to 2020, respectively. The decline in $\mathrm{H} 7 \mathrm{~N} 9$ was mainly due to the largescale vaccination of animals beginning in 2017, which led to a large reduction in human cases. These small and relatively stable diseases are not affected by other factors under their own strict prevention and control measures. We suggest that the only way to decrease the monthly incidence of these diseases may be to improve the prevention and control measures targeting these specific diseases. As for the impact of preventive measures on the monthly mortality of zoonotic diseases, no significant one was found in the emergency stage, as all protective measures that did not involve disease treatment, such as any restrictions of movement and wearing face masks during the COVID-19 emergency stage, had no effect on the pathogenic mechanism, pathological changes, clinical manifestations or outcomes of zoonotic diseases.

\section{Limitations}

The strengths of the present study include the analysis of national-level data covering all regions of mainland China, comprehensive examinations of the impacts of preventive measures during the COVID-19 emergency and routine stages on zoonotic diseases, and yield of sensible results. However, our study also has several limitations. First, to compare the annual incidence and mortality of zoonotic diseases in 2020 with those in 2015-2019, we calculated the average annual incidence and mortality from 2015 to 2019. Such pooling of data across time points may mask some variations in annual incidence and mortality. Nevertheless, this approach has been applied in some studies and has proven to be appropriate. ${ }^{29}{ }^{30}$ In their study, Lai et al concluded that a 5-year average incidence was more accurate when estimating the impact of the COVID-19 epidemic on other diseases due to the year-to-year fluctuation in relation to these data. ${ }^{31}$ Second, in 2020, we regarded January 2020 to April 2020 as the emergency stage of the COVID-19 pandemic and May 2020 to December 2020 as the routine stage. However, some provinces, such as Zhejiang province, relaxed the physical distancing measures earlier than other regions based on zero local case in the late emergency stage. Similarly, during the routine stage, there were also high-risk areas or communities with occasional virus transmission, such as the Xinfadi community in Beijing. These areas and communities underwent lockdown until there were no new cases for 14 days after confirmation of the last case. Nevertheless, in this study, our goal was to investigate the overall situation across China rather than in specific regions. It is more appropriate to consider most regions in China than specific localities. Third, as discussed above, the positive impacts of the incidence of zoonotic diseases may be the result of the combined effects of strict non-pharmaceutical measures and people's avoidance of 
seeking treatment from healthcare services. In this study, avoiding medical care was a major confounding factor that could not be avoided.

\section{CONCLUSION}

Strict containment and feasible suppression strategies during the emergency stage of the COVID-19 pandemicwith core measures including lockdown in endemic areas, travel restrictions and physical distancing-successfully stopped the spread of COVID-19 in China and had positive impacts on the incidence and mortality of zoonotic diseases, especially those with a relatively high number of annual cases. The main goal of the long-term mitigation strategy without physical distancing during the routine stage of the COVID-19 pandemic has been to maintain no or minimal local cases of COVID-19 until the population is protected through immunisation with safe and effective COVID-19 vaccines. Preventive measures such as wearing face masks during the routine stage had no significant effective impact on the incidence and mortality of zoonotic diseases.

In the future, zoonotic diseases will likely return to their long-term downward trend or to relatively stable seasonality. For zoonotic diseases, an effective strategy for lower incidence and mortality has been improving and completing the prevention and control measures that directly affect animals. It is necessary to emphasise the harmonious coexistence of humans and animals in nature, to develop new technologies for timely monitoring and detection of new pathogens that may spread to humans in order to quickly control them, to educate people on related health matters, to reduce damage to nature, to encourage people to seek medical treatment, to report suspected cases immediately and to train medical forces from rural areas to respond to such diseases. It is also necessary to strengthen the monitoring of personnel in key places, such as livestock settings, farmer's markets, flower and bird markets and breeding farms.

\section{Author affiliations \\ ${ }^{1}$ College of Statistics and Data Science, Faculty of Science, Beijing University of Technology, Beijing, China \\ ${ }^{2}$ Center for Applied Statistics, School of Statistics, Renmin University of China, Beijing, China \\ ${ }^{3}$ Department of Geriatrics, The First Affiliated Hospital, Zhejiang University School of Medicine, Hangzhou, Zhejiang, China \\ ${ }^{4}$ Nanjing Jiliang Information Technology, Nanjing, China \\ ${ }^{5}$ Department of Infectious Diseases, Zhejiang Provincial Center for Disease Control and Prevention, Hangzhou, Zhejiang, China}

Acknowledgements We thank the editor and reviewers for their careful review and insightful comments, which have led to a significant improvement of this article.

Contributors WX, SL and FL designed the study. CM and XG conducted the data analysis. CM, XG and LW drafted the manuscript. WL, WX and SL reviewed the manuscript. All authors read and approved the final version of the manuscript. WX is the guarantor of the study and had full access to the data.

Funding This work was supported by the Beijing Natural Science Foundation (Grant No Z200001), National Natural Science Foundation of China (Grant No 11971478), Public Health and Disease Control and Prevention, and the Major Innovation and Planning Interdisciplinary Platform for the 'Double-First Class'
Initiative in Renmin University of China (Grant No 2021PDPC), the Zhejiang Provincial Program for the Cultivation of High-Level Innovative Health Talents, the Medical and Health Research Project of Zhejiang Health Commission (Grant No 2020KY525), the Medical and Health Research Project of Zhejiang Health Commission (Grant No 2021 KY629), the Zhejiang Provincial Natural Science Foundation of China (Grant No GF21H260012), the Non-profit Central Research Institute Fund of Chinese Academy of Medical Sciences (Grant No 2020-PT330003), the Health Commission of Zhejiang Province, China (Grant No WKJ-ZJ-2106).

Competing interests None declared.

Patient consent for publication Not required.

Provenance and peer review Not commissioned; externally peer reviewed.

Data availability statement Data are available in a public, open access repository. Data are available in a public, open access repository. No additional data were available.

Supplemental material This content has been supplied by the author(s). It has not been vetted by BMJ Publishing Group Limited (BMJ) and may not have been peer-reviewed. Any opinions or recommendations discussed are solely those of the author(s) and are not endorsed by BMJ. BMJ disclaims all liability and responsibility arising from any reliance placed on the content. Where the content includes any translated material, BMJ does not warrant the accuracy and reliability of the translations (including but not limited to local regulations, clinical guidelines, terminology, drug names and drug dosages), and is not responsible for any error and/or omissions arising from translation and adaptation or otherwise.

Open access This is an open access article distributed in accordance with the Creative Commons Attribution Non Commercial (CC BY-NC 4.0) license, which permits others to distribute, remix, adapt, build upon this work non-commercially, and license their derivative works on different terms, provided the original work is properly cited, appropriate credit is given, any changes made indicated, and the use is non-commercial. See: http://creativecommons.org/licenses/by-nc/4.0/.

\section{ORCID iDs}

Chenjin Ma http://orcid.org/0000-0002-2483-2309

Wangli Xu http://orcid.org/0000-0003-4983-9950

\section{REFERENCES}

1 Malkhazova S, Mironova V, Shartova N. Mapping Russia's Natural Focal Diseases: History and Contemporary Approaches. Cham: Springer, 2018: 1-20.

2 Rahman MT, Sobur MA, Islam MS, et al. Zoonotic diseases: etiology, impact, and control. Microorganisms 2020;8:1405.

3 Al-Tayib OA. An overview of the most significant zoonotic viral pathogens transmitted from animal to human in Saudi Arabia. Pathogens 2019;8:25.

4 Taylor LH, Latham SM, Woolhouse ME. Risk factors for human disease emergence. Philos Trans $R$ Soc Lond B Biol Sci 2001;356:983-9.

5 Daszak P, Cunningham AA, Hyatt AD. Anthropogenic environmental change and the emergence of infectious diseases in wildlife. Acta Trop 2001;78:103-16.

6 Busani L, Platonov AE, Ergonul O, et al. How to tackle natural focal infections: from risk assessment to vaccination strategies. Adv Exp Med Biol 2017;972:7-16.

7 Chinese Center for Disease Control and Prevention. Annual report on notifiable infectious diseases, 2020. Available: http://www.nhc.gov. cn/jkj/s3578/202103/f1a448b7df7d4760976fea6d55834966.shtml

8 World Health Organization. WHO Director-General's opening remarks at the media briefing on COVID-19, 2020. Available: https://www. who.int/dg/speeches/detail/whodirector-general-s-opening-remarksat-the-media-briefing-on-covid19-11-march-2020

9 Kupferschmidt K, Wadman M. Delta variant triggers new phase in the pandemic. Science 2021;372:1375-6.

10 Di Renzo L, Gualtieri P, Pivari F, et al. Eating habits and lifestyle changes during COVID-19 lockdown: an Italian survey. J Trans/ Med 2020;18:229.

11 Xiang M, Zhang Z, Kuwahara K. Impact of COVID-19 pandemic on children and adolescents' lifestyle behavior larger than expected. Prog Cardiovasc Dis 2020;63:531-2.

12 Lee H, Lee H, Song K-H, et al. Impact of public health interventions on seasonal influenza activity during the SARS-CoV-2 outbreak in Korea. Clin Infect Dis 2021;73:e132-40.

13 Hsieh C-C, Lin C-H, Wang WYC, et al. The outcome and implications of public precautionary measures in Taiwan: declining 
respiratory disease cases in the COVID-19 pandemic. Int J Environ Res Public Health 2020;17:4877.

14 Olsen SJ, Azziz-Baumgartner E, Budd AP, et al. Decreased influenza activity during the COVID-19 pandemic-United States, Australia, Chile, and South Africa, 2020. Am J Transplant 2020;20:3681-5.

15 Soo RJJ, Chiew CJ, Ma S, et al. Decreased influenza incidence under COVID-19 control measures, Singapore. Emerg Infect Dis 2020;26:1933-5.

16 Gutman JR, Lucchi NW, Cantey PT, et al. Malaria and parasitic neglected tropical diseases: potential syndemics with COVID-19? Am J Trop Med Hyg 2020;103:572-7.

17 Hogan AB, Jewell BL, Sherrard-Smith E, et al. Potential impact of the COVID-19 pandemic on HIV, tuberculosis, and malaria in lowincome and middle-income countries: a modelling study. Lancet Glob Health 2020;8:e1132-41.

18 Lazzerini M, Barbi E, Apicella A, et al. Delayed access or provision of care in Italy resulting from fear of COVID-19. Lancet Child Adolesc Health 2020;4:e10-11.

19 Tam C-CF, Cheung K-S, Lam S, et al. Impact of coronavirus disease 2019 (COVID-19) outbreak on ST-segment-elevation myocardial infarction care in Hong Kong, China. Circ Cardiovasc Qual Outcomes 2020;13:e006631.

20 Hung KKC, Walline JH, Chan EYY, et al. Health service utilization in Hong Kong during the COVID-19 pandemic - a cross-sectional public survey. Int J Health Policy Manag 2020.

21 Xiao J, Dai J, Hu J, et al. Co-benefits of nonpharmaceutical intervention against COVID-19 on infectious diseases in China: a large population-based observational study. Lancet Reg Health West Pac 2021;17:100282.
22 Chinese Center for Disease Control and Prevention, National Health Commission of the People's Republic of China. Monthly report of notifiable infectious diseases. Available: http://www.nhc.gov.cn/jkj/ s2907/new list.shtml?tdsourcetag=s pcqq aiomsg

23 Chinese National Bureau of Statistics. China statistical yearbook, 2021. Available: http://wwwstatsgovcn/tjsj/ndsj/2021/indexchhtm

24 Noufaily A, Enki DG, Farrington $P$, et al. An improved algorithm for outbreak detection in multiple surveillance systems. Stat Med 2013;32:1206-22.

25 Shi J, Deng G, Ma S, et al. Rapid evolution of H7N9 highly pathogenic viruses that emerged in China in 2017. Cell Host Microbe 2018;24:e7:558-68.

26 Raynor B, Díaz EW, Shinnick J, et al. The impact of the COVID-19 pandemic on rabies reemergence in Latin America: the case of Arequipa, Peru. PLoS Negl Trop Dis 2021;15:e0009414.

27 Department of Health, Hong Kong. Guidelines on prevention of coronavirus disease 2019 (COVID-19) for the general public, 2020. Available: https://wwwchpgovhk/files/pdf/nid guideline general public_enpdf

28 World Health Organization. Coronavirus disease (COVID-19) advice for the public, 2020. Available: https://www.who.int/emergencies/ diseases/novel-coronavirus-2019/advice-for-public

29 Lee K-K, Lai C-C, Chao C-M, et al. Increase in sexually transmitted infection during the COVID-19 pandemic in Taiwan. J Eur Acad Dermatol Venereol 2021;35:e171-2.

30 Fang J-L, Chao C-M, Tang H-J. The impact of COVID-19 on the diagnosis of TB in Taiwan. Int J Tuberc Lung Dis 2020;24:1321-2.

31 Lai C-C, Chen S-Y, Yen M-Y, et al. The impact of the coronavirus disease 2019 epidemic on notifiable infectious diseases in Taiwan: a database analysis. Travel Med Infect Dis 2021;40:101997. 